\title{
Old age as an experience in everyday life: Health and subjectivity in the voice of the old Basis for rethinking and reformulating social and government policies regarding the aging process
}

\author{
DOI: $10.46932 / \mathrm{sfjdv} 3 \mathrm{n} 1-053$
}

Received in: Jan 30st, 2021

Accepted in: Feb 1th, 2022

\author{
Norma González González \\ Full-time research professor at the Faculty of Political and Social Sciences \\ Autonomous University of the State of Mexico UAEMex. Country. Mexico. \\ E-mail: gogn@uaemex.mx / gogn66@yahoo.com.mx
}

\begin{abstract}
In today's societies, the growth of the older adult population has become a problem, and in a particular way it is presented as a challenge in terms of public policies. It has been common for attention to be directed to the needs of a medical-pharmacological nature and the interest it represents in demographic terms, presenting it as a serious problem for which contemporary societies are not prepared. However, it is a phenomenon that must be recovered and thought about in the mosaic of a complexity that forces us to direct our gaze towards other references and dimensions that turn out to be central in the claim for their comprehensive attention. Recover the first person speech of those who experience old age; their concerns, tensions, fears, needs and material and emotional requirements, entails and commits actions that in the short and medium term can make a difference in the face of a problem that is urgent to make visible, giving it existence in its different nuances and dimensions, in order to be able to generate the necessary interventions.
\end{abstract}

Keywords: Health and old age, Old age and subjectivity, Medicalization, Public health.

\section{PRESENTATION}

In Mexico and in the world, the increase in the older adult population has gradually become a problem for society in general, and in particular a challenge in terms of public policies. It has been common for attention to be directed primarily to the needs of a medical-pharmacological nature and the interest it represents in demographic terms, as part of a model of society for which, at the time, the increase in life expectancy was raised as one of its main flags around the idea of progress and development. However, now that the increase in the aging population has become a reality, it is presented as a serious problem for which contemporary societies are not prepared in terms of the care and provision of goods and services that are required and they are assumed to be financially and economically unviable.

In terms of public policies, the way in which the government constructs and carries out its decisions at the federal and local levels (Aguilar, 1994), represents in this case a central place for the definition of strategies of attention, care and follow-up to this population group that, in addition to assuming a socio- 
epidemiological and demographic concern, must be recovered and thought about the mosaic of complexity that forces us to direct our gaze towards other references and dimensions that turn out to be central in the claim of comprehensive care for old age. The aging process that Mexican society is going through requires knowledge and recognition of a phenomenon in issues that have been invisible and alien both in terms of government actions and of interest, concern and social mobilization; remaining outside the discussion and academic and social knowledge. The subjective experience of old age places the phenomenon in a comprehensive care plan that calls for the involvement of the government and society in their different expressions and interactions in areas as representative as the family, the community, the school, and society all civil.

It is urgent to recover the first-person discourse of those who experience old age; their concerns, tensions, fears, needs and material and emotional requirements, and before which society as a whole seems alien and indifferent. Knowing, commits actions that in the short and medium term can make a difference in problems such as the one referred to here, a necessary first step is to give visibility to a phenomenon to give it existence, and thus be able to generate the necessary interventions.

Indeed, the approach to old age involves different areas and levels of expression and social representation; Doing so from a qualitative perspective contributes to making visible a series of aspects and conditions that have traditionally been alien to research interests both from the biomedical perspective and in the field of social sciences (González, 2005). Which has implications in the field of the social as well as in that of public policies, fundamentally oriented to those spaces that dominate a collective imagination regarding old age and its reduced needs and limited to the socio-demographic interest and the biomedical and economic field.

It has been common to approach the phenomenon from a quantitative perspective, in such a way that the wear and tear and organic deterioration that the epidemiological data collect has left aside a fundamental aspect that worsens over time and that should be recognized as one of the the most important references for the care of the elderly; that is, everything related to emotional management at a stage of life in which the need for dialogue, conversation, exchange of ideas, a place and a space where fears, anguishes, memories, unresolved duels, can be released, be heard, find an echo among peers, or in the attentive listening of professionals sensitive to this process; It is in this sense that the approach to subjectivity acquires a particular relevance and meaning.

\subsection{OLD AGE, HEALTH AND SUBJECTIVITY}

Medical science, heir to positivist thinking and ideals around the neutrality and objectivity of the so-called scientific method, in its daily practice straddles the so-called exact sciences and the field of 
social sciences, which has given way to a controversy in force today, regarding its scope and limitations in health care. The knowledge achieved by the social sciences, lets us see that science is not a faithful and objective reflection of reality, but is a complex network of interpretations and meanings that result from human activity in society. It is in this sense that the exclusion of subjectivity in the field of health, its care, and particularly the care of the disease in the different stages of life, constitutes a great deficiency that reduces and limits the possibilities of knowledge in this field, as in that of social and public actions aimed at attending to the vast mosaic of needs and requirements that the field of health involves, and even more so when it is linked to one of the stages of life such as, in this case, old age. People, individuals, whether women or men, are more than organs, systems, and functions, consigned and structured in the manner of biological bodies. Of course, it is impossible to depart from the biological connotation, but this only acquires meaning and significance in the symbolic and emotional representation of our daily reality (Ackerknecht, 1985)

Throughout our individual and collective existence, based on the values, the meanings that guide our actions, relationships and social interactions, we experience life in diverse and complex ways according to age, social class, our occupations or professions, the level of education, ethnicity or social group to which one belongs, etc; In this sense, the body lives and expresses in a particular way the experience of what is called health and disease. Old age constitutes one of those stages of life that, in recent decades, has been occupying an increasingly prominent place in the context of social problems that demand attention and monitoring in governmental and social activities, hence the importance of make subjectivity one of the main axes to approach old age, overcoming its dominant connotations that have limited other access routes that go beyond its mere description and epidemiological and demographic monitoring, opening channels of knowledge that place us in a greater proximity and empathy of this complex process of which we are part both directly and indirectly.

Everything that has to do with and builds subjectivity, structures what we know as social life, where the articulation of the varied and diverse discursive forms build and give meaning to the social order. In the understanding that it is from social subjects and their interactions that the conditions and factors (economic, political, social and cultural) are continually elaborated, produced and reproduced on which life in society is built. That is to say, that the actions of the subjects take place "acquires" meaning in the fabric of social relationships and processes, since subjective actions in their daily processes and interactions make what we call social reality possible.

At present, the strengthening of the approaches and theories that make the presence and importance of the subject and subjectivity more and more visible in the approach to social reality and its growing complexity, has placed the social sciences as the ideal arena in which takes place a whole series of debates 
and theoretical and methodological proposals that contribute to the discussion in favor of the subjective plane as an essential component in the construction of knowledge, and whose importance and origin is found in the very conformation of reality social (Wolf, 1988; Alexander, 2000). In the particular case of the relationship between old age and health, any claim in terms of government and public policies or social actions in general is impossible, if old age and health are not assumed in terms of complex processes that involve experience daily life of social subjects (Curcio et al, 2022), demanding a comprehensive approach to their diversity, and the possibilities of governmental and social intervention.

\subsection{GENERAL DEMOGRAPHIC OVERVIEW}

In modern societies, aging is the result of a downward shift in mortality and birth rates, to a pattern of lower and controlled levels of mortality and birth rates, a phenomenon that is recognized as a demographic transition whereby so-called developed societies have gone through, and through which population changes take place in the so-called developing countries under their own population references. In Mexico, according to the National Institute of Statistics and Geography (INEGI, 2019), the demographic transition began in the 1930s with the fall in mortality and thirty years later, in the 1960s with the downward modification of the rate of fertility, which over time has led to the widening of the tip of the pyramid where the older population is located, and where the growth of the older adult population is graphically represented.

Continuing with the information provided by INEGI (2019), we find that in the 1930s, life expectancy was 33 years for men and almost 35 years for women, while in the first decade In the 21 st century, life expectancy had moved to 71 and 77 years, respectively for men and women. Likewise, according to the most current data, for 2016, the figures are expressed as follows: 72.6 and 77.8 , for men and women.

These figures also lead us to point out a phenomenon called feminization of old age, characterized by the fact that worldwide, and Mexico is no exception, the older adult population is made up mostly of women, which translates into and means that women live longer than men. According to information provided by INEGI, in 1990, in the population group aged 60 and over, the male / female ratio was 89 men for every 100 women. If the information is disaggregated into different age groups aged 60 and over, the difference is more noticeable starting from the group aged 85 and over, where the ratio is 73.8 men for every 100 women, the feminization of aging here reaches its peak higher figure, as women register the longest life expectancy (INEGI, 2014). 


\subsection{IN SEARCH OF A DEFINITION}

In the eighties, the World Health Organization WHO, proposed the concept of "older adult" in reference to people of sixty years and over (WHO, 2021), trying to face the growing use of pejorative language in the face of the process aging; Even then, the contemptuous treatment of a population group that was becoming numerically more and more significant in the different countries and regions of the world was evident. It was in 1996, when the United Nations organization (UN, 2021), based on a chronological-demographic reference, established the age of sixty years as the criterion to define and group the population considered as the elderly population, a criterion that in Mexico is recovered through the National Institute of Older Adults (INAPAM, 2019), in the Law of the Rights of Older Adults, and also in the Official Mexican Standard NOM-167-SSA-1997, for the purposes of the provision of health care services carried out by the Ministry of Health SSA, to provide monitoring and services to this population group.

Currently, in Mexico, at the institutional level, INAPAM is responsible for formulating and implementing programs and actions aimed at this sector of the population; However, it should be noted that the complexity of the phenomenon demands inter-institutional efforts and civil society in areas such as the economy, health, education, social support, capable of facing the diversity and complexity of a phenomenon that supposes a called in terms of public policies, but perhaps more important, is the fact of socially rethinking a sense and responsibility towards life that, in personal and family spaces (in the broadest and most open sense of the new models and concepts of family) generate and introduce a culture of respect and care for old age, in general respect and recognition of each of the stages of life, paying for a respectful coexistence; dignified for a population group that forces us to keep in mind a moment of existence that awaits us; Of course, if it is possible to overcome the accumulation of risks that today represent a threat at increasingly early ages: accidents, disease, poverty, wars, violence in its different expressions and dimensions, etc., and with the scope and power to truncate life before reaching old age.

\subsection{OLD AGE ITS SOCIAL HISTORICAL CONSTRUCTION}

In each culture and historical moment, old age assumes a symbolic representation, inserted in the power relations of which it is a part. Rosen mentions that “... the stages of the life cycle not only depend on physiological maturity but, and above all, on the way in which society recognizes, defines and structures such stages in terms of social roles and attitudes" (Rosen, 1985: 60). In the logic of other cultures and / or historical moments, old age embodies wisdom, and gives rise to rituals marked by acts and shows of respect, recognition of authority. What does not happen in the framework of a neoliberal and globalized society where old age is viewed with contempt, disgust and indifference, assuming it as a 
burden that must be disposed of in its unproductive condition, a condition (this, that of unproductiveness) high and clearly penalized in today's society, where everything is measured and moves based on productivity, consumption capacity, financial utility (González, 2018; El País, 2013; Bauman, 2013)

Posing old age as a social construction implies assuming that beyond the biological approaches that normalize ages, that is, the different periods of life in terms of childhood, youth, adulthood and old age, resorting at each stage to traits that seem naturally immanent to them, the fact is questioned that although it is impossible to separate the biological from the social, it is important and indispensable to place the biological and the social in the field of their own cultural and historical horizons and scopes (INMUJER, 2015; Butler, 1999). In this sense, we speak of the ages of life as a social construction in reference to the fact that each of these periods, of these stages (are generally accepted as a process immanent to life but that historically they have not always existed as they do today) we know), to which roles and roles are assigned, which regulate our social activities, our behaviors (Kehl and Fernández, 2001). On a biological basis, the different ages of life and their representations are a function of culture (Ackerknecht, 1985), and it is essential that the reference society (s) sanction them as such, to plunge their existence into terms of childhood, youth, adulthood, old age, shared by an imaginary that in the course of social processes and relationships, makes it one of the most important axes for the conformation and construction of identities, which give life and meaning to the relationships and interactions, making these roles compatible in terms of places, functions, and representations that, socially, we have of each of the different stages in the course of life.

\subsection{OLD AGE AS A PROBLEM?}

Faced with a powerful discourse that exalts youth and reviles old age, societies must face a series of institutional and social demands that make it possible to face a phenomenon that demands health services: specialized medical care, medicines, medical instruments, diagnostic services, hospital infrastructure, hospital beds, etc; while in the socioeconomic sphere there is a reorganization of family and community life in order to establish the spaces, the processes, the type of relationships that correspond to this phenomenon that is today part of our daily reality; also, in a context of Mexican society in which new values and sociocultural references make it symbolic and practically different from other moments in the history of the country. What do we do with (our) old people? What is their role and place in the current family and societies? How do we organize ourselves to make them participate in a coexistence in which now they are the ones who require care and time ?, (time) today is such a scarce resource to communicate, in addition to the fact that in the case of younger generations, the skills, mechanisms and traditional ways to carry out interaction with their peers have been lost (Contino, 2014; Kravetz, 2013), 
these are new realities of communication and social interaction that in general tend to be alien to the elderly population.

Especially in the second half of the last century, the phenomenon of living longer is closely related to the improvement of what was called living conditions, that is; an economic and social development that particularly favors the implementation of health measures (which were intended to encompass and involve the diagnosis, treatment and prevention of diseases). It was assumed that the conditions of food, personal and collective hygiene, the working conditions, among the most outstanding, as a whole were translated into a greater number of years lived. However, in a prominent way so far this century, an attempt has been made to establish a difference between the increasing number of years lived (demographers name this phenomenon an increase in life expectancy) and its difference with a concept more complex and controversial as is the quality of life in old age. In this sense, it is not just about living longer, but that this lengthening of life occurs in a favorable environment, both material and warm and human support; which unfortunately does not happen neither in our country, nor in the global world. In this sense, the phenomenon entails a challenge that, in terms of public policies, must generate responses to the requirements of health, participation and economic and social inclusion (Muñoz, 2011; Robles et al, 2008). While it is true that we are living longer and longer, it is also true that in our societies it is very frowned upon, and the fact of aging is stigmatized, which contrasts with an exacerbated cult of youth.

In this direction, it would be necessary to question whether old age is automatically equivalent to disability and dependency, or if it is the social context in which we live, which provides the references to think about it and assume it in the way in which it has become a reality load. It is necessary to ask why for our society, old age, the aging process becomes a problem, when even in the middle of the last century the increase in life expectancy in so-called developed societies was seen as one of the great achievements of development and the progress of the western world, of the so-called modernity (Robledo et al, 2015). The old are no longer productive, they no longer consume, then they have lost their economic value, they are something that is left over, that bothers, that bothers us, that in the unconscious reminds us of the rejection of the "destiny" that awaits us, and far from a With respectful recognition of our own journey through life and into old age, we denigrate them as if it could change the path of the end of our life. In the hedonistic, narcissistic and individualistic society that dominates us, old age and death have become a taboo subject (González, 2018; Bazo, 1998; Lipovetsky, 1998)

\section{METHODOLOGY}

On the theoretical basis of an approach and a look that tries to question and show the stigma that for modern societies old age represents as the last stage of life, this document is the result of a documentary 
work that incorporates the most current critical discussion in around aging, and the urgent need to recover first-hand the experience of those who face this process in its daily complexity. Thus, from a qualitative approach, part of the information obtained in the framework of a series of in-depth interviews and participant observation is recovered, carried out with older adults throughout 2019, as well as part of the field work developed that same year in the shelter called "Casa de día", dependent on the Institute of Social Security and Services for State Workers ISSSTE, and located in the city of Toluca. In both cases, participant observation and in-depth interviews became the tools used to obtain information about the experience of older adults regarding their own aging process, closely linked to their daily living spaces and relationships the family and, where appropriate, coexistence in the care center called "Casa de día".

\subsection{MEDICALIZATION OF OLD AGE AND DEATH. OLD AGE AND DEATH AS A DISEASE?}

In this direction, a key concept is that of medicalization, understood as “ ... the process by which certain phenomena that initially form part of other fields such as education, law, religion, etc; they have been redefined as medical phenomena. The process refers to the way in which the realm of modern medicine has expanded in recent years, and now encompasses many problems that were not previously considered medical entities; including a range of manifestations, such as the normal phases of the productive cycle (menstrual cycle, pregnancy, childbirth, menopause), unhappiness, loneliness, baldness, of course old age and death (Fuster et al, 2016). The critical thinking that arises around the medicalization process forces us to think that more than drugs, we need to go through other care channels that can significantly affect the improvement of the conditions and quality of life of older adults. Old age is not a disease, so it cannot be automatically synonymous with medical and pharmacological care (Ociel, 2013), but with a current involvement and commitment at different levels, spaces and scales of social coexistence that recognizes and does Their demands for integration and social coexistence are evident, having as axes the respect and dignity of people in each and every stage of life.

The medicalization of the different processes and stages of life is a reality that, however, on a dayto-day basis we are unable to perceive and identify as such. The normalized presence of medicine in everyday life runs through the most personal acts of our lives. In the particular case of "the end of life", medical and pharmacological interventions assume one of their most recurrent expressions, identifying old age with the invariable need for medical and pharmacological interventions. Against the current, it is said that in various directions and senses old age, as part of a natural process on the path of life, is capable of facing with its own resources some of its physiological and organic "discomforts" typical of the wear of organs, body and cognitive functions; However, the need for pharmacological medical attention and intervention is automatically assumed, making the lack of communication, of interaction of the elderly 
with a social and family environment that is increasingly hostile to them, invisible, where slowness does not seem to fit of their movements and their speech, where their opinions and points of view are ignored and a source of annoyance for their environment, representing the context that aggravates and in many cases is the origin of the processes and conditions of discomfort, illness, deficiencies in which old age is lived and experienced (Robledo et al, 2015). From children, through the different processes of socialization that begins in the family and continues in school, we learn to visualize old age as an unwanted stage of life, hence the rejection, the alien and indifferent meaning that we socially show To her.

When the elderly interact in a favorable family and social environment, or as part of institutional support groups, as is the case of the Day House referred to here; there where coexistence between older adults is favored (sharing concerns, joys, hopes and expectations in life), and where there is also the respectful, kind, gentle and professional care of workers trained to understand and attend to their needs, not solely or exclusively medical; and where you can also participate in activities that favor the preservation of physical and cognitive health: making crafts, playing a musical instrument, participating in painting, reading, writing groups, etc., it makes them feel useful, creative, in an environment where the group and coexistence with their peers makes a difference (Juul, 2015)

\subsection{OLD AGE AS AN EXPERIENCE}

In macro-social terms, old age usually refers to demographic data or morbidities that are assumed to be typical of this moment in life. The experience of being old has gone to the background, or has definitely been erased from any attempt to learn about the phenomenon and, where appropriate, generate intervention actions. However, as has been insisted in this document, knowing and knowing about old age from subjectivity contributes to opening our focus of attention, revealing aspects and dimensions that contribute to generating integral actions of attention and care, with the capacity to significantly improve the quality of life of the elderly, thereby laying the foundations of what will be our own aging process.

\footnotetext{
"... Since I came to this group, I have someone to talk about my sorrows with, and also those who take care of us do so with affection and respect... everyone works in my house, I don't even exist there..." (UAEMEX, 2019A).

"Here I come and my ills are gone... I have made new friends... we share what happens to us and when we can, we help each other..." (UAEMEX, 2019A).
}

Living with those we consider to be our peers, to the extent that they are assumed to be those who share our experiences, and can understand what happens to us, are recognized with a therapeutic power that relieves and cures.

Indeed, one of the elements that stand out in coexistence in specialized care centers, as referred to here, has to do with the interaction and daily care that makes us feel alive and important to an environment 
that is representative of us, which contrasts with the indifference that old people live in a family space where they are no longer visible and important, and it seems that nobody cares and interests what they feel and think, what they want to share, and from the outside it is assumed as insignificant and typical of age.

\footnotetext{
"The forces diminish, they go; the body no longer responds as before. People, your same family begins to see you differently..." (UAEMEX, 2019B).

"Your hands are wrinkled, your face... you see it every day there is no turning back, it is very difficult, very hard to accept it...". (UAEMEX, 2019B).

"For me it was something that came suddenly; that one pain, that another, I was left without work, I went into a strong depression... I have been more than a year and I am not recovering, that is why they recommended me to come here, where there are others already old like me ... "(UAEMEX, 2019B).
}

The internal and external bodily transformations that accompany old age place in a situation of "crisis" those who must face changes that seem to distance them from activities, tasks, and above all from daily coexistence; the same labor participation that no longer recognizes them as viable to perform as someone of a younger age can ideally do. Likewise, old age implies a variable and difficult mental process, of accommodations and resignations that entails emotional states that have remained oblivious to the attention and care that this stage of life demands. The bodily changes in which old age is recognized represent one of the greatest stigmas of our societies where the cult of eternal youth dominates.

\subsection{THE ARRIVAL OF DEATH}

Old age, in some ways, is usually accompanied by a greater sense of closeness to death. This is closer and it is known that it awaits us. However, the type of society in which we live today has distanced us from it, in such a way that we do not know how to address it and manage its proximity; something strange because as they say colloquially, death is the only sure thing we have in life.

"You do not make plans, you know that there is no time, a feeling of sadness comes to you, that you did not know, it is a new feeling, your family does not understand it..." (UAEMEX, 2019A).

"... Of course I think of death, I feel it close, and the unknown scares me" (UAEMEX, 2019B).

In the past, the arrival of death occurred in the family home, with the accompaniment of loved ones and in spaces where part of the existence of the dying person was recognized, today death occurs in the loneliness and coldness of hospitals. What on the other hand, represents a practical and very useful mechanism for the relatives, who are responsible for collecting the body of the deceased, giving way to the today indispensable and lucrative work of funeral homes so that, in the shortest possible time, the relatives can They recover the normality of life, return to work and the normality of the family. The hectic, individualistic, hedonistic and narcissistic life no longer allows us other types of rituals before death. 
"I am afraid of dying in a hospital but I know that this is my destiny" (UAEMEX, 2019B).

"I ask God one day to wake up dead, so as not to nag my children" (UAEMEX, 2019A).

"I ask a lot, but I don't want to be taken to the hospital, I find it very sad to die there" (UAEMEX, 2019A).

In the so-called developed countries, where the demographic and epidemiological transition occurred in anticipation of the so-called underdeveloped world, hospitals are overwhelmed by the demand for care of dying patients of advanced ages who die in them, a fact for which hospital professionals health and hospitals themselves were not prepared. This phenomenon begins to be recurrent in societies such as Mexico.

\subsection{THE SOCIAL CONSTRUCTION OF OLD AGE IS ALSO A QUESTION OF GENDER}

Gender is a category that brings us closer to understanding the social, historical, political and cultural construction of sexual difference, in such a way that both men and women are the result of a long and complex process of assigning roles that stereotype our doing social throughout life. The socialization process that begins in the family and continues in school, gives way to the generation of an identity subjected to a set of socio-cultural conditioning, determining roles and roles of men and women. Indeed, in the different spaces where socialization takes place and comes to life, values, behaviors, expectations are produced and reproduced, on which behavior, actions and social phenomena are founded and acquire meaning. During childhood we are taught norms, values, and ways of conducting ourselves in particular contexts. This process holds the keys to looking at and understanding the world, its relationships and interactions; in such a way that old age, its representations and meanings, are built in a daily flow that from the beginning of life shapes and fixes ideas and images around it (Keijzer, 20199). Socializing agents, in addition to the family, are of course the school, the community, friends, and work spaces; Today the media with their technological developments to which the emergence of omni present social networks is tied, are in charge of maintaining and reproducing gender roles.

As a social construction, gender cuts across all areas of social relations and processes. In the socialization process that begins in childhood within the family, and continues in school, the differences that build us as men or as women in the performance of gender roles that define our behaviors and interactions are defined and structured social. Over time, in the course of their lives, men and women are traversed by different states and conditions of vulnerability that respond to the socioeconomic context of ascription and the type of institutional protection available (Salgado, 2007; Sabo, 2000)

\footnotetext{
"They recommended this care home to me, my problem, well I see it as a problem is that there are only women..." (UAEMEX, 2019A).
} 
Until recently, when talking about gender, it seemed that the allusion was only to what had to do with the conditions of subordination and inequality in which women have survived, however in recent years there is an increasing concern and interest to make visible and make known the experiences that give shape and meaning to being a man, and that also represents risk conditions or lack of care derived from their own condition, subject to rules and forms of coexistence that limit their participation or that imply a particular form of experiences in life as in this case, in the ways in which old age is experienced. The study of the so-called masculinities, understood as “... the set of attributes, values, behaviors and behaviors that are characteristic of men in a given society" (CNDH, 2021: 1), however in the same document it is noted that "Currently the presence of a universal man is questioned, since acting as a man varies according to the historical, social, cultural context, etc. "(CNDH, 2021: 1); has favored the power to unveil a series of realities where everyday existence is expressed and makes sense, an ordinary world that conforms to norms, to rules that define and limit the senses as a provision of an order that guides our actions, relationships and interactions, as the basis for the production and reproduction of life in society.

\footnotetext{
"When my wife lived, my daughters took her there with her family. I'm still at home, I'm going to die there..."(UAEMEX, 2019A).

"... I can no longer work, I have supported myself all my life, and I will not be waiting for someone to give me money, my children have their own problems..." (UAEMEX, 2019A).

"It's all over now, my self-esteem is on the ground, without work, without how to support myself, only, I know what I live and no one else"... "(UAEMEX, 2019B).
}

The experience of old age is different for men and women, as it is the product of particular ways of assuming life, their roles, their roles, their identities. The family is the first space where these differences are present. Women, as mothers, tend to maintain a greater relationship and contact with their children until the end, especially with their daughters, so they can be incorporated into their respective families or they tend to spend periods of time caring for these daughters and their families. In the case of men, coexistence is more distant, and it is very difficult for a man to accept living and taking care of his children, he prefers to stay at home alone. Life as a provider took him away from coexistence and contact with his children, so this is reflected in this last stage of life. In their role as providers, men lose their worth by not being able to continue working and being independent, they feel diminished before themselves and before others.

The fact that in places of care such as the Day Home that is recovered here, it is predominantly women who go to this type of care spaces, accounts for the particular ways of aging among men and women. Women usually demand more attention without feeling shame or shame, they are not as limited as in the case of men to show weakness, and demand care. It is not strange in this sense that very few men go to these care centers where it is difficult for them to demand attention and care; It is not strange in this sense to be convinced that there is no one with whom to share and coexist, given that "there are only 
women", who in principle are assumed as different, not as peers, with whom to share what they live and feel ; This is a clear expression of that lack of communication between the genders that has accompanied us all our lives and that achieves one of its expressions in the discourses of older adults.

\subsection{THE OLD IN THE CONTEXT OF THE PANDEMIC}

We cannot end this document without making a reference, albeit a general one, to what the pandemic can mean for a group of the population that is traditionally assumed to be vulnerable. The Covid-19 pandemic that we suffer today and affects us globally, also represents a problem with many edges and that involves different levels of social reality, so it is important to investigate and know what happens in a group of a population that, it has been recognized, is one of the most affected by both their living conditions and the type of care and attention they demand. In Europe, at the beginning of the pandemic, the highest number of deaths was concentrated in nursing homes and care homes for the elderly; the phenomenon with its peculiarities and differences seems to affect this age group that is traditionally assumed to be one of those with the greatest risk and vulnerability. In the case of Mexico, the official information reported by the Secretary of Health SSA, and is presented every day since the declaration in the country of the first deaths from covid-19, and where updated information is exposed day by day, highlights the reference to those over 60 years of age, usually linked to serious problems of comorbidities

Beyond the importance that undoubtedly this type of epidemiological monitoring has, it is necessary and important to recover other planes and aspects of old age, which refer us to a daily life in the basic sense of the production of life in society. Hence the need to ask, for example, what happens to older adults in the framework of this global health phenomenon, how they live and experience a situation of these dimensions and characteristics on a day-to-day basis; Before the pandemic, reference was already made to the condition of loneliness and abandonment that they suffered within families, an easy conjecture would be to think that they are now accompanied at home, however this is not necessarily the case. For example, within the framework of gender studies and mobilizations, it is said that confinement has exacerbated and diversified the physical and emotional violence that is exercised towards them within the family space, in this logic it is important to look at what It happens with the elderly, really before the permanent presence in the home of those who used to be absent to go to work or school, loneliness and the different types of violence that this population group had been suffering, or as in the In the previous example, the mere physical presence of the family members does not translate into accompaniment and communication, and if on the contrary it has given rise to other types of actions and attitudes that affect the elderly within the families. It is undoubtedly a pending work that will have to be addressed in the short term, information is required that brings us closer to the life experiences of the elderly, to move towards 
a greater commitment and awareness as a society, and of course to be able to implement public actions that give follow-up and attention to this social phenomenon that must involve each and every one of us, regardless of our age. The youngest of us forget that, for better or for worse, the world we inhabit is the product of the work carried out by the generation that precedes us.

\section{BY WAY OF CONCLUSIONS}

It is urgent and necessary to open the discussion and debate aimed at generating actions that involve and commit us in the strengthening of a culture of aging; the one that has to do, yes with institutional programs but also with daily actions and attitudes of respect, accompaniment and solidarity; vindicating the conscience and responsibility that each generation has regarding the actions and criteria for which we are jointly responsible for the dignity of each of the stages of life (SSA, 2015b; Muñoz, 2011; Robles, 2008)

Investigating, knowing about old age, translates into a mosaic of interests and complex and diverse searches that demand greater responsibility and collective sensitivity, capable of permeating and transforming daily life. As mentioned at the beginning of this document, socially and academically we must move towards other types of knowledge with the ability to identify, and where appropriate generate actions that collect the complexity of social needs and problems from places where the subject assumes its leading role. Only in this way, in the field of public policies, will it be possible to face the need and interest in discussing, defining and, where appropriate, designing actions and programs that deal with a phenomenon that is assumed to be a problem of economic, social and cultural dimensions. As Eduardo Ibarra mentions, a cult of the pragmatism of the administration has dominated, the exaltation of its power and its technical efficiency, its apparent neutrality, which has marginalized its approach as a social practice (Ibarra, 1998), which always ends up subordinate to the dominant interests that in each historical moment mark the route and the modalities in which it must be incorporated to give body to the governmental action.

The active participation of the population is undoubtedly required to achieve important changes that involve the commitment of the different social sectors without which it is impossible to achieve significant changes to ensure that old age can be synonymous with dignity. The phenomenon that we are currently experiencing at the global level represents a clear example that without the participation of all it will be impossible to achieve favorable results for intervention in any type of phenomenon that involves and defines us as a society. The phenomenon to which we refer here, transcends the field of medicine, and places us at an unprecedented crossroads, but where this is noticed in what this small text strives to show: the aging process, is first of all a phenomenon of a social nature, and should be treated in this way from 
governmental instances, thus guaranteeing its best management and approach in terms of governance. Knowing about a process or phenomenon compromises our individual, collective and institutional performance. 


\section{BIBLIOGRAPHY}

Ackerknecht, Erwin H (1985) Medicine and social anthropology. AKAL / University. Madrid Spain-

Aguilar, Villanueva Luis F (1994) The study of public policies. Introductory study. Porrúa editors. Mexico.

Alexander, Jeffrey C. (2000) Cultural Sociology. Forms of classification in complex societies. ANTHROPOS. Spain.

Bauman, Zygmunt (2013) Culture in the world of liquid modernity. FCE. Mexico.

Bazo, María Teresa (1998) Dependent old age, policies and quality of life "in Papers 56, pp. 143-161. University of the Basque Country, Spain.d

Butler, Judith (1999) The Gender in Dispute. Paidos.

Colomer, Revuelta Concha (2007) "The sex of the indicators and the gender of inequalities" Editorial. in Spanish Journal of Public Health 81: 91-93. Spain.

Contino, Alejandro Martín (2014) "Biopolitics and public policies in vulnerable population sectors" in Perspectives of Public Policies Year 4 No. 7 July-December, pp. 105-125

CNDH (National Human Rights Commission) (2021) Respect for different masculinities. Because there are many ways to be a man. CNDH. Mexico. https://www.cndh.org.mx/sites/all/doc/Programas/Ninez_familia/Material/trip-respeto-difmasculinidades.pdf. Accessed March 25, 2021.

Curcio, Carmen Lucia et al (2022) “Asociación entre espacio de vida y factores psicológicos y funcionales en personas mayores de Brasil y Colombia" en South Florida Journal of Development, V.3 N. 1 jan-feb, pp. 252-270. DOI: https://doi.org/10.46932/sfjdv3n1-020

Fuster, Sánchez Nicolás and Moscoso-Flores, Pedro (2016) "Power at the time of the population. Foucault and the medicalization of the modern city"in Athenea Digital Magazine 16 (3): 207-227.

González, González Norma (Coord) (2007) Poverty and health in the State of Mexico. Non-hospital diabetes care. Porrúa / UAEMEX / CEMAPEM. Mexico

González, González Norma (2018) "Medicalization of death. Elements of discussion and analysis for a critical approach from the social sciences". Cultural Magazine. Vol.6, pp. 1-20.

Ibarra Colado, Eduardo, "Administration and organization at the end of the millennium: rationalism, irrationalism and governmentality", in: Ramírez, Grajeda Beatriz (Coord.) The role of the administrator in the current context, Mexico City, UAM-A, year 1998, 186 pages.

INEGI (National Institute of Statistics and Geography) (2019) Tell me. Population of Mexico. Information by

http://cuentame.inegi.org.mx/monografias/informacion/mex/poblacion/default.aspx?tema=me\&e=15.

Accessed June 10, 2019.

INEGI (National Institute of Statistics and Geography) (2014) Sociodemographic profile of older adults. INEGI. Mexico. 
INMUJERES (National Institute of Women) (2015) Situation of older adults in Mexico. Statistics Directorate. Government of the republic / INMUJERES. www.inmujeres.gob.mx

INAPAM (National Institute for Older Adults) (2019) https://www.gob.mx/inapam. https://www.gob.mx/inapam. Retrieved May 7, 2019.

Juul, Lassen Aske (2015) "Biopolitics of old age. How knowledge about aging forms active aging policies" in Historical Sociology. Monograph Biopolitics and Social Sciences. Editions of the University of Murcia, Spain.

Kehl, Wiebel Susana and Fernández, Fernández J. Manuel (2001) "The social construction of old age" in Cuadernos de trabajo social 14: pp. 125-161.

Keijzer, Benno (2019) As far as the body can endure: Gender, body and men's health. http://www.estudiosmasculinidades.buap.mx/paginas/reporteBenodekeijzer.htm. Accessed October 29, 2019.

Kravetz, Tatiana (2013) "Old age and the new roles of the old in modern capitalist society" Paper presented at X Conference on Sociology of the UBA. Table: aging and society, pp. 1-8, Buenos Aires, Argentina.

Lipovetsky, Gilles (1998) The era of emptiness. Essays about contemporary individualism. Anagrama Editions. Barcelona, Spain.

Muñoz, Hernández Roxana (2011a) "The public policies of old age in Mexico 2010" in Iztapalapa Journal of social sciences and humanities, no. 71, July-December, pp. 35-60. Mexico.

Muñoz, Franco Nora Eugenia (2009b) "Reflections on self-care as a category of analysis in health" in the Salud Colectiva Magazine 5 (3): 391-401, Buenos Aires, Argentina.

Ociel, Moya Mario (2013) "Genealogy of an unannounced old age: biopolitics of aging bodies or the advent of gerontogovernmentality" in Revista Polis 36

Robles, Silva Leticia et al (2008) Caring for the elderly: evaluations around non-family care "in Revista Text @ Contexto Enfermagem Vol17 No., April -June, pp. 225-231, Brazil.

WHO (2021) World Health Organization. Aging and health. https://www.who.int/es/news-room/factsheets/detail/envejecimiento-y-salud.

UN (United Nations Organization (2021) Aging. Https://www.un.org/es/sections/issuesdepth/ageing/index.html. Accessed March 10, 2021.

Robledo, Gutiérrez Luis Miguel; Kershenobich, Stalnikowitz (Coord) (2015) “Aging and health. A proposal for an action plan. Third edition, UNAM. Mexico,

Rosen, George (1985) From Medical Police to Social Medicine. XXI century. Mexico.

Sabo, Don (2000) Understanding men's health. A relational and gender-sensitive approach. Harvard Center for population and development studies. OPS. USES.

Salgado, Nelly and Wong, Rebeca (2007) "Gender and poverty: determinants of health in old age" in Revista Salud Pública de México year / vol. 49, number Su4. National Institute of Public Health, pp. $515-$ 521. Mexico. 
SSA (Secretary of Health) (1999) OFFICIAL MEXICAN NOM-167-SSA1-1997, FOR THE PROVISION OF SOCIAL ASSISTANCE SERVICES FOR MINORS AND OLDER ADULTS. Published in the Official Gazette of the Federation on November 17, 1999 SSA. Mexico

SSA (Secretary of Health) (2015a) Health record. Report on the health of Mexicans. Undersecretary of Integration and Development of the Health Sector. SSA. Government of Mexico.

SSA (Secretary of Health) (2015b) General diagnosis of population health. Undersecretary of Integration and Development of the Health Sector. SSA. Government of Mexico.

UAEMEX (Autonomous University of the State of Mexico) (2019a) Interviews conducted at a Day Home dependent on ISSTE, located in Toluca; as part of the research project Aging and Human Rights.

UAEMEX (Autonomous University of the State of Mexico) (2019b) Interviews conducted in the second semester of 2019; as part of the research project Health, subjectivity and old age.

Wolf, Mauro (1988) Sociologies of everyday life. CHAIR. Madrid Spain.

\section{JOURNALISTIC NOTE}

El País (2013) "Hurry up and die" January 22. Madrid Spain. Accessed September 3, 2013. https://elpais.com/sociedad/2013/01/22/actualidad/1358865219_550162.html 